\title{
MODELING OF DIFFUSION AND PREMIXED FLAME OF AP/HTPB LAMINATE PROPELLANT
}

\author{
F. Maggi, L. T. DeLuca, G. Colombo, A. Bandera, \\ and L. Galfetti
}

\begin{abstract}
Composite propellants feature a diffusion flame. The size of oxidizer particles leverage some combustion properties (mainly, burning rate and pressure sensitivity) along with flame structure. Macroscopic combustion features are strictly related to those events occurring inside the gas phase and close to the burning surface. The flame of nonaluminized composite energetic materials is considered and a simplified combustion model is tested for this case. Combustion of a laminate propellant with varying lamina size is simulated. The benchmark consists of some movies taken from ammonium perchlorate (AP) / hydroxyl-terminated polybutadiene (HTPB) propellant combustion with a high-speed video camera. Three different powder sizes are used in propellant manufacturing.
\end{abstract}

\section{INTRODUCTION}

The flame of a composite propellant is a complex dynamic system that encompasses heat transfer, chemistry, chemical diffusion, etc. Its nature is strictly related to the features of the condensed phase. An AP-based propellant is a multiphase system. This material has an intrinsic heterogeneous nature from a microscopic point of view.

The simplest composition contains AP powder and a polymeric binder like HTPB. Ammonium perchlorate decomposes into oxidizing gases while binder pyrolysis produces fuel gases. In addition, a propellant can contain some metal powder as a fuel (like aluminum) and other minor additives like iron oxide, ammonium bichromate, etc.

Studies of flame structure were mainly focused on nonaluminized propellants because of their simplicity. Being without a metal phase, they are free from agglomeration issues and modeling complexity is by far reduced. Nowadays, nonaluminized propellants still play an important role in this field of investigation. 
This simple configuration features a complicated flame structure. Ammonium perchlorate decomposes into a reactive mixture. A premixed flame partly consumes the mixture if pressure is above 20 bar [1]. This reaction is mainly driven by kinetics. Combustion products are hot and still reactive [2]. Pure AP cannot sustain a deflagration wave when the pressure is set below 20 bar. When a propellant burns, AP decomposition products interact also with light-weight hydrocarbon gases from binder pyrolysis [3]. The resulting mixture is highly flammable also under subatmospheric conditions. This flame is diffusive. Moreover, random AP displacement inside the polymer matrix yields to unsteady flame structure. In fact, surface composition locally changes during combustion and the flame is effectively nonstationary, but only from a microscopic point of view.

Plenty of models were developed throughout the years. One can group them into two categories: one-dimensional (1D) and multidimensional. Some comprehensive summaries about 1D models can be found in [4-6]. Worth mentioning are the Granular Diffusion Flame model by Summerfield [7], the Hermance model [8] (with a statistic treatment of the oxidizer size), and the Beckstead-Derr-Price (BDP) model [9] (combustion driven by multiple flames).

All these models solve quickly a set of $1 \mathrm{D}$ equations. Heterogeneity treatment, if any, is mainly included by means of simple statistical tools. Several developments and refinements can be found in the literature as well. Nowadays, 1D models are still used to give rough estimates or when a submodel is required.

New efforts are focused on the multidimensional approach to the problem. Several two- (2D) and three-dimensional (3D) solvers fully dealing with heterogeneity have been worked out in the last few years.

Worth mentioning is a paper by Jackson and Buckmaster [10] which is the recapitulation of former papers dealing with a $3 \mathrm{D}$ combustion solver. The model includes 3D fluid dynamics and a simple two-step combustion scheme. Moreover, a packing algorithm can be used to model the heterogeneity of the solid phase. Combustion results can be used also as subgrid model in a full-scale rocket simulation [11]. More 2D combustion solvers are present in the literature as well [12-14]. They have lower requirements in terms of memory and computational resources but more limited capabilities. Nevertheless, if a complex 3D solver is not required, they allow to focus on some specific aspects of combustion.

The present work focuses on nonaluminized rocket propellant flame structure. Of particular interest is the effect of particle size. The capability of capturing the arising physical changes by a simple $2 \mathrm{D}$ combustion model is tested. At this stage of the work, the verification is mainly qualitative and consists of some comparisons between numerical simulations and real flame images. Combustion movies are taken with a high-speed video system. Attention will be given to the heterogeneity of the gas phase. 


\section{FLAME AND NUMERICAL MODEL DESCRIPTION}

A comprehensive description of nonaluminized propellant flame is given by Beckstead et al. [9] and the explanatory scheme is shown in Fig. 1. Beckstead model states the presence of a premixed flame settling just above the AP crystal due to its oxidizing decomposition products. A primary diffusion flame is present alongside the AP particle for some of the oxidizing gases diffuse into the binder decomposition products. This flame does not consume all reactive components. Finally, leftovers of premixed and primary diffusion reactions mix altogether and generate a final diffusion flame. Gas stream is still laminar as reported by Steinz et al. [7] and also visible in Fig. 1 (snapshot of a propellant combustion with AP size $250 \mu \mathrm{m}$ at 5 bar).

The present combustion model consists of a basic set of $2 \mathrm{D}$ equations. It was initially presented by Miccio [12]. Some parameters were modified in former works $[2,15]$ but the governing equations are essentially the same (for a sake of brevity, a reader is referred to those papers for the full description of the code):

$$
\frac{\partial M_{g} C_{g}}{\partial t}+\frac{\partial}{\partial x}\left(M_{g} C_{g} u_{x}\right)=0
$$

where $M_{g}$ is the molar mass of the gas phase which is assumed uniform and constant, $C_{g}$ is the molar density, and $u_{x}$ is the gas velocity along $x$ direction;

$$
\frac{\partial Y_{i}}{\partial t}=-u_{x} \frac{\partial Y_{i}}{\partial x}+\frac{\partial}{\partial x}\left(D \frac{\partial Y_{i}}{\partial x}\right)+\frac{\partial}{\partial y}\left(D \frac{\partial Y_{i}}{\partial y}\right)+G_{i}
$$

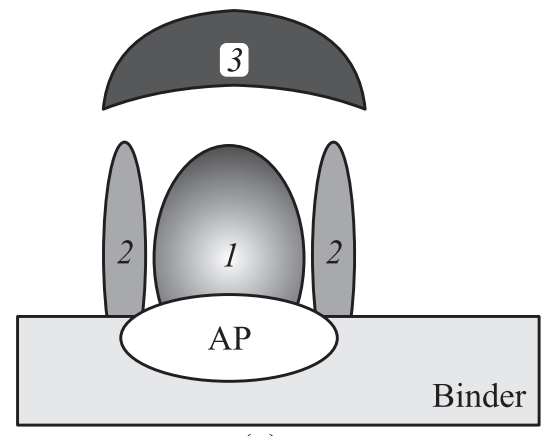

(a)

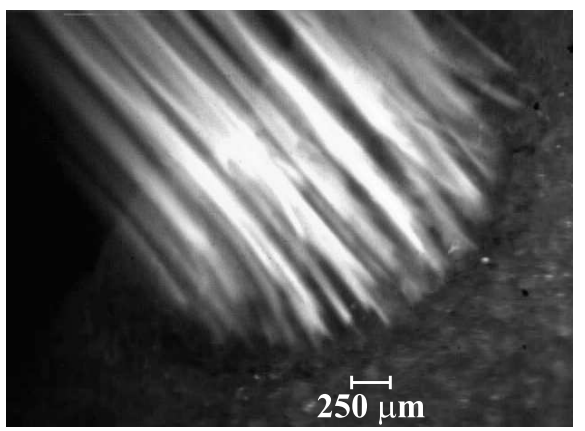

(b)

Figure 1 Multiple flame model $(a)$ and propellant combustion at 5 bar $(b)$ : $1-$ premixed flame; 2 - primary diffusion flame; and 3 - final diffusion flame 
where $Y_{i}$ is the molar fraction of the $i$ th species, $D \propto\left(T^{7 / 4} p^{-1}\right)$ is the mass diffusivity, and $G_{i}$ is the source term for the $i$ th species;

$$
\frac{\partial T}{\partial t}=\frac{\partial}{\partial x}\left(\alpha_{g} \frac{\partial T}{\partial x}\right)+\frac{\partial}{\partial y}\left(\alpha_{g} \frac{\partial T}{\partial y}\right)-u_{x} \frac{\partial T}{\partial x}-\frac{1}{C_{g} \gamma_{g}}\left(r_{4} \Delta h_{4}+r_{5} \Delta h_{5}\right)
$$

where $T$ is the temperature, $\mathrm{K}, \gamma_{g}=\left(27+0.013 T-2 \cdot 10^{-6} T^{2}\right)$ is the quadratic interpolating polynomial for the specific heat, $\mathrm{J} / \mathrm{mol} / \mathrm{K}, \alpha_{g}$ is the thermal diffusivity evaluated in each grid point, $\Delta h_{i}$ is the enthalpy of the $i$ th reaction which evolves with the rate $r_{i}$, and gas conductivity is assumed constant;

$$
C_{g}=\frac{p}{\Re T}
$$

where $p$ is the pressure [16] and $\Re$ is the universal constant of gas, $\mathrm{J} / \mathrm{mol} / \mathrm{K}$; and

$$
\frac{\partial T}{\partial t}=\frac{\partial}{\partial x} \alpha_{s} \frac{\partial T}{\partial x}
$$

Thus, the fluid is treated in the model as inviscid. The flow is treated as laminar and 1D motion equations (1) are adopted. Chemical species conservation and heat balance are governed by $1 \mathrm{D}$ transport and $2 \mathrm{D}$ diffusion models of Eqs. (2) and (3). The gas phase is treated as a perfect gas according to Eq. (4). Heat transfer is computed inside the solid phase only along $x$ direction according to Eq. (5). Diffusivity of the solid phase is set constant in $x$ direction when laminate configuration is adopted. The initial propellant maintains both chemical and physical heterogeneity. Thus, burning surface composition can change in space and time reflecting the displacement of reactants in the bulk. Reactivity is confined by the gas-solid interface and is present in the gas domain while the solid bulk is nonreactive.

The following five overall reactions set up the reactivity in the gas phase and the regression rate of the condensed matter:

$$
\begin{array}{ll}
\mathrm{R} 1: A_{s} \rightarrow 45 B_{g} & r_{\mathrm{R} 1}=A_{1} \exp \left(\frac{-E_{a(1)}}{\Re T_{s}}\right) ; \\
\mathrm{R} 2: C_{s} \rightarrow D_{g}+E_{g} & r_{\mathrm{R} 2}=A_{2} \exp \left(\frac{-E_{a(2)}}{\Re T_{s}}\right) ; \\
\mathrm{R} 3: \frac{1}{5} A_{s}+E_{g} \rightarrow 4 F_{g} & r_{\mathrm{R} 3}=A_{3} Y_{E} \frac{p}{\Re T} \exp \left(\frac{-E_{a(3)}}{\Re T_{s}}\right) ; \\
\mathrm{R} 4: D_{g}+\frac{1}{2} E_{g} \rightarrow 2 F_{g} & r_{\mathrm{R} 4}=A_{4} p^{1.8} Y_{D} Y_{E} \exp \left(\frac{-E_{a(4)}}{\Re T}\right) ; \\
\mathrm{R} 5: B_{g}+\frac{1}{2} E_{g} \rightarrow 2 F_{g} & r_{\mathrm{R} 5}=A_{5} p^{1.5} Y_{B} Y_{E} \exp \left(\frac{-E_{a(5)}}{\Re T}\right)
\end{array}
$$




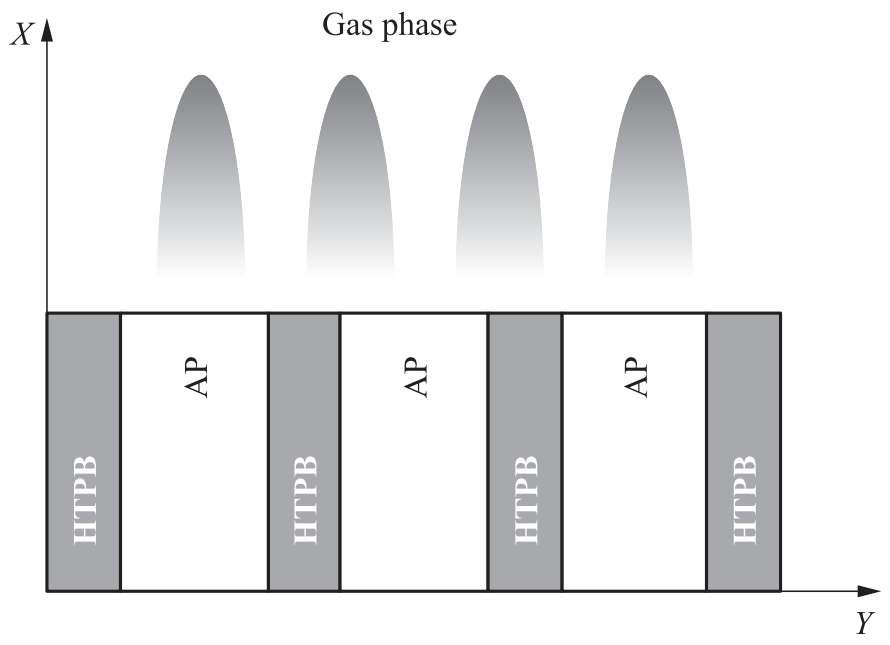

Figure 2 Computational domain. Laminate propellant and axes orientation

where $E_{a(i)}$ is the activation energy and $A_{i}$ is the preexponential factor. The component $A$ represents the binder; component $C$ is the oxidizer; $B, D$, and $E$ are the intermediate products; and $F$ is the final species.

This reaction scheme is by far simpler than fully resolved kinetics but it captures some essential aspects of the combustion process. Equations (6) and (7) represent the decomposition of binder and oxidizer. Equation (8) is a heterogeneous reaction between solid binder and oxidizing gas species. Equation (9) is the premixed combustion and Eq. (10) traces all the diffusive reactions.

The $2 \mathrm{D}$ computational domain is plotted in Fig. 2 together with axes orientation. Laminate propellant configuration is chosen to get rid of unsteadiness inside the flame structure. The use of a random AP displacement in the bulk would get a more representative propellant but surface composition would change during combustion. Keeping the AP/HTPB mass ratio of $80 / 20$, the lamina thickness is varied. Note that this mass ratio is not valid for single crystal combustion tests.

Boundary conditions consist of zero derivatives on sides as well as on top of the domain for all variables. Ambient temperature is set at the bottom of the solid phase. The gas-solid interface moves over because of the material consumption. Regression rate is treated according to the local composition by Eqs. (6) and (7). Those reactions also assign the boundary condition for the chemical composition of the gas phase. One more condition is set for gas velocity: 


$$
u_{x(\mathrm{loc})}=\frac{\rho_{s(\mathrm{loc})} r_{b(\mathrm{loc})}}{\rho_{g(\mathrm{loc})}}
$$

where $\rho_{s(\text { loc })}$ is the local solid density; $r_{b(\text { loc })}$ is the local consumption rate of the solid phase; and $\rho_{g \text { (loc) }}$ is the gas density at the surface temperature as comes from the equation of state.

The thermal balance across the gas-solid interface includes surface reactions:

$$
k_{g} \frac{\partial T}{\partial n}=k_{s} \frac{\partial T}{\partial n}-\left(r_{\mathrm{R} 1} \Delta h_{1}+r_{\mathrm{R} 2} \Delta h_{2}+r_{\mathrm{R} 3} \Delta h_{3}\right)
$$

where $k_{s}$ and $k_{g}$ are the thermal conductivities of solid and gas phase, respectively.

\section{REAL PROPELLANT COMBUSTION}

Some nonaluminized propellants with different powder size $\phi$ were investigated with a high-speed video recording technique. The AP/HTPB mass ratio for each of those propellants was set to $80 / 20$. There was no provision for any additive for compatibility or burning catalysts. Each propellant contained one of the following powder fractions:

- fine fraction: $\phi_{f}=5-10 \mu \mathrm{m}$;

- intermediate fraction: $\phi_{m}=80-140 \mu \mathrm{m}$; and

- coarse fraction: $\phi_{c} \approx 400 \mu \mathrm{m}$.

Selection of $\phi_{m}$ was done with calibrated sieves whereas $\phi_{c}$ and $\phi_{f}$ came from an industrial supplier.

Combustion tests were run inside a horizontal bomb filled with nitrogen at a preset pressure in the range 1-15 bar. The steel container had a volume of two liters and had two windows placed on the sides of the sample. The third window was located at the rear end of the chamber for laser beam inlet. The facility comprised a laser source and a controlled exhaust system. Acting as nonintrusive heating source, the laser beam granted the ignition while a set of automatic valves kept the pressure inside a narrow range even during combustion.

The camera was placed on one side together with a long range microscope and a cold light source. The absence of metal led to darker flame with respect to metalized formulations. Thus, frame rate had to be limited to 500 frames per second for the sake of visibility. 


\section{FRAMES FROM EXPERIMENTAL TESTS}

In the following pictures, some frames taken from propellant combustion movies are shown. Figures 3 and 4 are related to the operating pressure of 1 bar while Figs. $5 a$ to $5 c$ are related to the 15 -bar combustion. As one comes from a coarser AP fraction (Figs. $3 b$ and $5 c$ ) to the finer one (Figs. $3 a$ and $5 a$ ), the flame becomes more regular and uniform. The combustion of propellants containing coarse AP at 1 bar features long and separate flames. Conversely, fine AP grants a well-mixed reaction field.

Figure 4 magnifies one single flame from coarse-AP propellant. Flame dimension is close to the crystal size, so one may argue that it may spring from one single particle. Similar discussion can be done for combustion at higher pressure

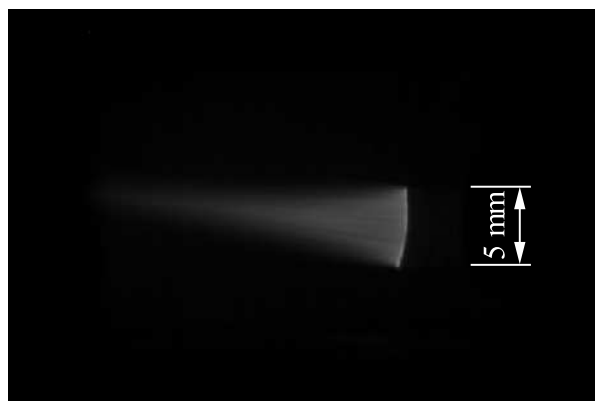

(a)

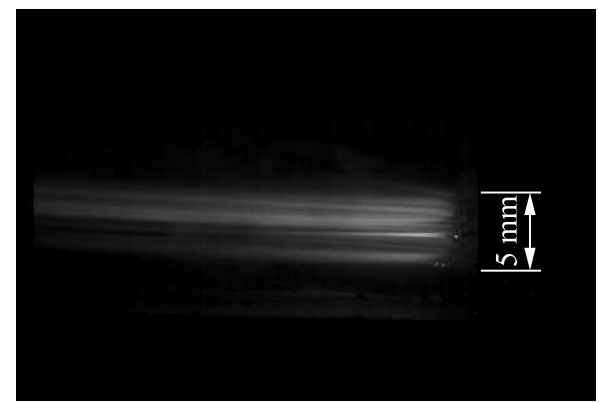

(b)

Figure 3 Propellant with fine AP size $\phi_{f}(a)$ and course AP size $\phi_{c}(b)$. Combustion at 1 bar

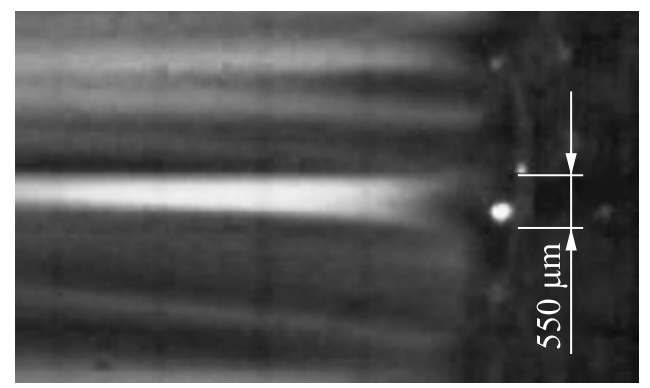

Figure 4 Detail of propellant combustion with course AP size $\phi_{c}$ at the operating pressure of 1 bar 


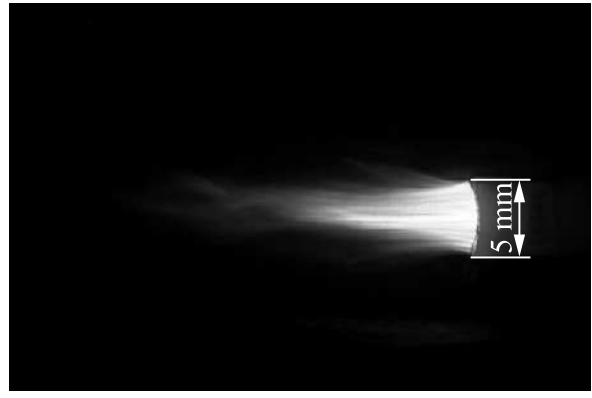

(a)

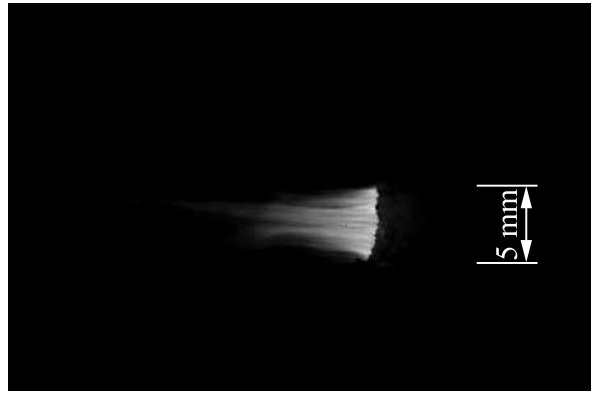

(b)

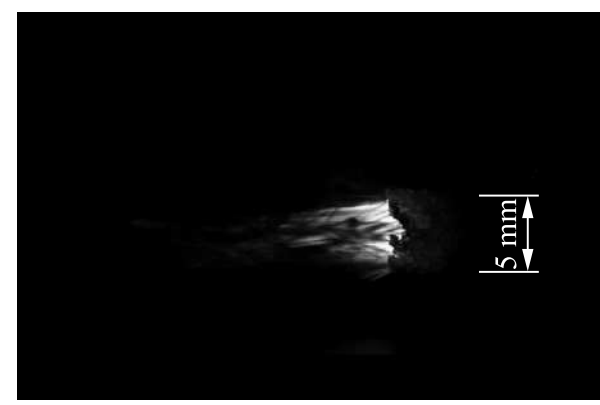

(c)

Figure 5 Propellant with fine AP size $\phi_{f}(a)$, intermediate AP size $\phi_{m}(b)$, and coarse AP size $\phi_{c}(c)$ at 15 bar

though the overall flame length is reduced in all cases. Besides, the process is faster and it becomes difficult to get clear and detailed images.

\section{APPLICATION OF COMBUSTION MODEL}

In this section, the model of Section 2 is applied to some laminate propellant configurations (Figs. 6 and 7).

Combustion is performed at 60 bar with three different AP sizes: laminas of 56 and $112 \mu \mathrm{m}$ (Fig. 6) and a crystal of $400 \mu \mathrm{m}$ (Fig. 7). Incorrect burning rate predictions still required some tuning of chemistry; mainly, by setting up a proper pressure dependence. Also, the premixed flame is not well resolved by the spatial discretization in use and this could impair the rate prediction capability. 


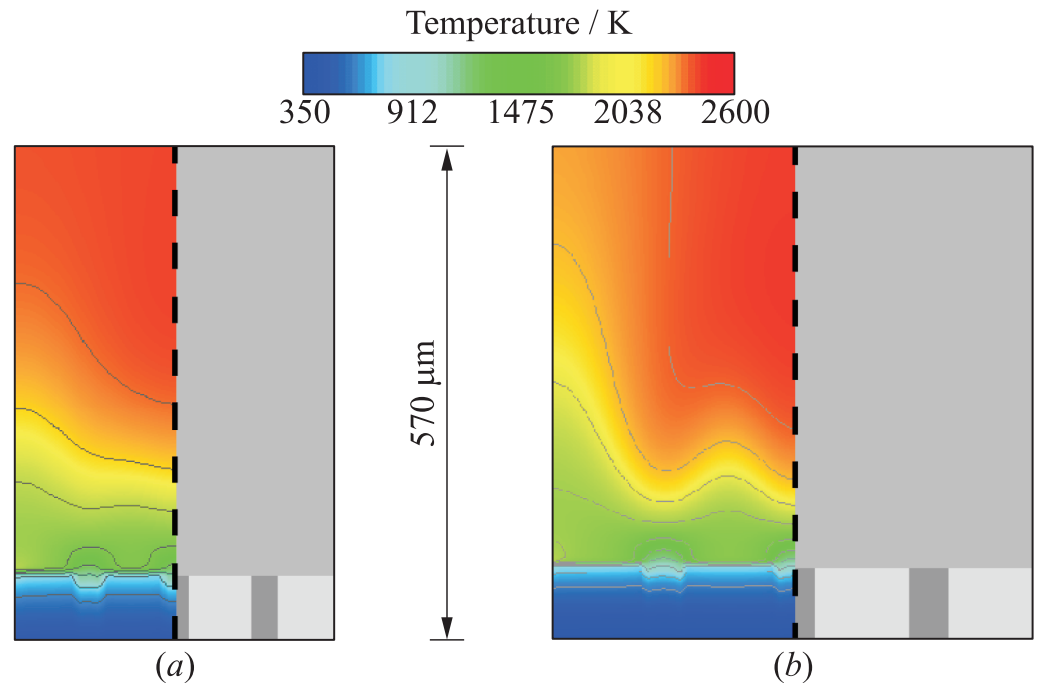

Figure 6 Combustion of AP/HTPB laminas. Each figure is split along the symmetry plane into temperature field (left) and solid phase map (right): (a) AP laminas size is $56 \mu \mathrm{m}$ and (b) AP laminas size is $112 \mu \mathrm{m}$. Combustion pressure is 60 bar. (Refer Maggi et al., p. 117.)

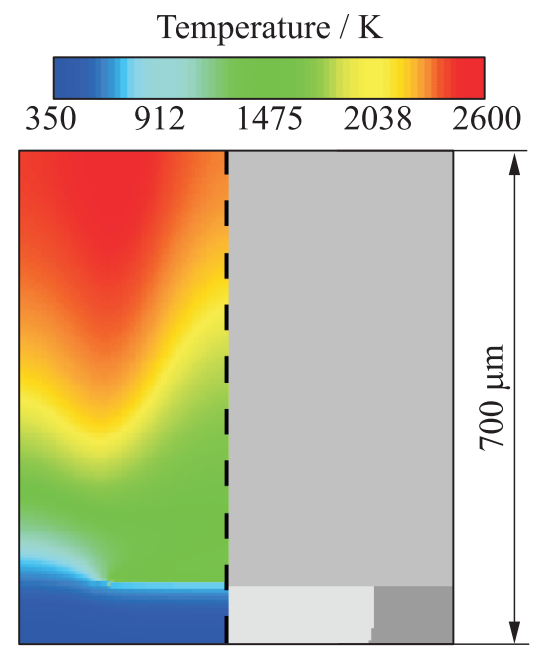

Figure 7 Combustion of an AP crystal of size $400 \mu \mathrm{m}$ embedded in HTPB. The figure is split along the symmetry plane into temperature field (left) and solid phase map (right). Combustion pressure is 60 bar. (Refer Maggi et al., p. 117.) 
Conversely, the physical model describes the main features of the diffusion flame and the correspondence between temperature fields and flame visualization is good. Simulation with coarser AP represents a heterogeneous flame.

The use of 400-micron AP (Fig. 7) produces a structure close to the experimental view of Fig. 4. In this case, side diffusion of reactants is slow and does not allow a proper mixing close to the burning surface. Flame structure is stretched along the stream direction. The primary diffusion flame is also present. The final diffusion flame is weak. Experimental pictures suggest that the completion of the final flame occurs far from the burning surface.

Finer laminas enhance mixing capability (Fig. 6). Diffusion blends reactants closer to the gas-solid interface (far enough from the side boundary). The final flame approaches the burning surface as the AP lamina size is decreased. Heterogeneity of the solid phase reduces its influence on the gas phase and the temperature field appears to be uniform above the final diffusion flame. The primary diffusion and premixed flames are too short to be observed clearly in the experiments. Moreover, the use of very fine AP tends to produce a flat final flame. In this case, reactant diffusion might be fast enough to homogenize the gas phase prior to any reaction and the premixed flame can occur. The experimental representation of this case is given in Fig. $3 a$.

Combustion movies at higher pressure depict the same trend, albeit it is not as evident as for the experiments at 1 bar.

\section{CONCLUDING REMARKS}

Experimental and modeling activities were performed on nonaluminized rocket propellants. The work was focused on the shape and inner structure of the flame. Some propellants were manufactured and combustion movies were taken. Laminate propellant configuration was numerically tested with a simplified combustion model. The influence of the oxidizing particle size was traced with qualitative observations and both experiments and modeling agreed on the flame structure modifications.

This work tested the capability of the simplified model originally suggested by Miccio [12] to catch the essentials of the combustion process. Yet, some refinements are needed in the chemical scheme and in the spatial discretization close to the burning surface to ensure better prediction capabilities of macroscopic combustion features like the burning rate.

As particle size was reduced, a progressive enhancement in mixing was observed in the gas phase. A trend to homogenize gaseous reactants prior to any reaction was identified. In this case, the upcoming flame may turn to premixed but further experiments and simulations are needed to better analyze this issue. 
These conclusions are valid for propellants ranging in normal rocket operating conditions (namely, up to 100 bar). Above this range, the models based on multiple flames tend to cease (e.g., Summerfield's GDF [7]) and the AP regression rate does not follow a potential trend with pressure [1]. In addition, the use of finer AP progressively yields to a higher pressure exponent in the Vieille's law and to combustion problems such as extinction or intermittent burning in some pressure range [7]. All these features may be correlated to a modification in the combustion process (namely, kinetics, diffusion, etc.) and some refinements should be introduced in the model to capture those behaviors.

\section{ACKNOWLEDGMENTS}

The authors wish to express their appreciation to Dr. Francesco Miccio for the helpful discussions regarding the combustion model.

\section{REFERENCES}

1. Boggs, T.L. 1970. Deflagration rate, surface structure and subsurface profile of self-deflagrating single crystals of ammonium perchlorate. AIAA J. 8(5):867-73.

2. Maggi, F., L. Galfetti, F. Miccio, and L. T. DeLuca. 2006. Flame structure simulation of nonaluminized composite propellants. 57th International Astronautical Conference Proceedings. Valencia, Spain, No. IAC-06-C4.P.3.03. 1-14. IAF.

3. Arisawa, H., and T. B. Brill. 1996. Flash pyrolysis of hydroxyl-terminated polybutadiene (HTPB) I: Analysis and implications of the gaseous products. Combust. Flame 106(1-2):131-43.

4. Kishore, K. 1979. Comprehensive view of the combustion models of composite solid propellants. AIAA J. 17(11):1216-24.

5. Cohen, N.S. 1979. Review of composite propellant burn rate modeling. AIAA J. 18(3):277-93.

6. Ramohalli, K.N.R. 1984. Steady state burning of composite propellants under zero cross-flow situation. In: Fundamental of solid propellant combustion. Eds. K. K. Kuo and M. Summerfield. Progress in astronautics and aeronautics ser. New York, N.Y.: AIAA Inc. 90:409-77.

7. Steinz, J. A., P. L. Stang, and M. Summerfield. 1969. The burning mechanism of ammonium perchlorate-based composite solid propellants. Technical Report 830 . Princeton University, Department of Aerospace and Mechanical Sciences.

8. Hermance, C.E. 1966. A model of composite propellant combustion including surface heterogeneity and heat generation. AIAA J. 9(4):1629-37. 
9. Beckstead, M. W., R. L. Derr, and C. F. Price. 1970. A model of composite solidpropellant combustion based on multiple flames. AIAA J. 8(12):2200-7.

10. Jackson, T. L., and J. Buckmaster. 2002. Heterogeneous propellant combustion. AIAA J. 40(6):1122-30.

11. Massa, L., L. T. Jackson, and J. Buckmaster. 2004. Using heterogeneous propellant burning simulations as subgrid components of rocket simulations. AIAA J. 42(9):1889-900.

12. Miccio, F. 1998. Numerical modeling of composite propellant combustion. 27th International Symposium on Combustion Proceedings. The Combustion Institute. 2387-95.

13. Cazan, R., and S. Menon. 2003. Direct numerical simulation of sandwich and random-packed propellant. AIAA Paper No. 2003-5082.

14. Groult, S., and A. Bizot. 2004. Numerical simulation of heterogeneous AP-HTPB propellant combustion. AIAA Paper No. 2004-4039.

15. Maggi, F., F. Miccio, and L. T. DeLuca. 2005. Effects of heterogeneity on flame structure of AP-HTPB propellants. European Conference of Aero-Space Sciences. Moscow, Russia.

16. Clavin, P. 1994. Premixed combustion and gasdynamics. Annu. Rev. Fluid Mech. $26: 321-52$. 